\title{
Gestión del proceso de supervisión docente en el colegio Dr. Clodomiro Picado Twight, Circuito 02 de la Dirección Regional de Educación de Turrialba y Jiménez, para la evaluación del desempeño docente
}

\author{
Evaluating Faculty Performance through a Supervisory Management Process at the Dr. Clodomiro Picado Twight High School in \\ Costa Rica's Circuit 02 of the Turrialba and Jimenez School District \\ Sirleny Ramírez Méndez ${ }^{1}$ \\ Ministerio de Educación Pública \\ Universidad de Costa Rica \\ Cartago, Costa Rica \\ sirle506@yahoo.es
}

Recibido: 04 junio 2015 • Aceptado: 04 febrero 2016 • Corregido: 18 febrero 2016

\begin{abstract}
Resumen. El presente artículo corresponde a un estudio sobre los enfoques de supervisión presentes en la gestión educativa; sus estrategias, factores positivos que se derivan de dicho proceso, así como las limitaciones que enfrentan quienes realizan esta función en los centros educativos y que inciden en la calidad educativa. La investigación se realizó en el Colegio Dr. Clodomiro Picado Twight de Turrialba y su propósito es analizar la gestión del proceso de supervisión docente en la institución, con el fin de establecer una propuesta para la evaluación del desempeño docente. El estudio es de carácter descriptivo, el cual muestra una incongruencia en la aplicación de un enfoque específico de supervisión, la necesidad de implementar más estrategias de supervisión docente, así como minimizar el impacto del factor limitante que obstaculiza los procesos de supervisión en la organización educativa. La propuesta de intervención se orienta a guiar la gestión, a ejercer un enfoque de supervisión democrática y participativa que considera al personal docente, administrativo y estudiantes en este proceso.
\end{abstract}

Palabras clave. Educación; administración; administración de la educación; gestión de la educación; supervisión en educación; supervisión docente; estrategias de supervisión; enfoques de supervisión docente; desempeño docente

\begin{abstract}
This article is based on research pertaining to supervisory approaches for educational management, its strategies, positive factors resulting from the process and constraints faced by those execute these roles in schools and how it impacts quality of education. This research study was conducted at the Dr. Clodomiro Picado Twight High school in Turrialba and is based on the objective of establishing a proposal for supervising the teaching staff. Findings are descriptive and reflect inconsistencies in using a specific supervisory. One need is to use more strategies for teacher
\end{abstract}

1 Bachiller en la Enseñanza del Inglés, Universidad de Costa Rica. Licenciada en Enseñanza del Inglés, Universidad Americana. Master en Administración Educativa, Universidad de Costa Rica. Actualmente labora como docente de inglés en el Colegio Ambientalista de Pejibaye e imparte cursos en la Universidad de Costa Rica, Turrialba. Anteriormente, se desempeñó como docente de inglés en el Colegio Técnico Profesional de la Suiza, Turrialba, y como facilitadora de cursos de aprovechamiento para el Colegio de Licenciados y Profesores en Letras, Filosofía, Ciencias y Artes (COLYPRO). 
supervision and minimize the impact of negative factors that limit supervision of educational institutions. The conclusion suggests an intervention approach to guide the principal in exercising a democratic and participatory approach to supervision including teachers, administrative staff and students.

Keywords. Education, school administration; education management; supervision in education; teacher supervision; supervision strategies; teacher supervision approaches; teaching performance

\section{Introducción}

El objetivo principal del estudio se orienta en concientizar a los gestores de la educación, personal docente y estudiantes, sobre la importancia de ejercer la supervisión en los centros educativos desde la gestión, en procura de mejorar los procesos internos y por ende, la calidad educativa. Esto obedece a políticas internacionales de la Organización de las Naciones Unidas para la Educación, la Ciencia y la Cultura (UNESCO) o de la Organización de Estados Iberoamericanos (OEI) que repercuten, directamente, en el sistema educativo costarricense. Por lo cual, el Ministerio de Educación Pública (MEP) crea políticas como el Centro educativo de calidad como eje de la educación costarricense y las 10 Líneas Estratégicas; ambos documentos tienen como meta final, lograr un cambio significativo en la calidad del sistema educativo público costarricense; cambio que va desde la gestión que se realiza en cada organización escolar hasta los procesos pedagógicos desarrollados en las aulas.

De acuerdo con el nivel jerárquico, la calidad educativa depende de quienes planifican, supervisan, controlan, evalúan y corrigen los procesos que se llevan a cabo en cada institución, de los gestores educativos y de las autoridades ministeriales. A nivel de centro educativo, la calidad de la educación requiere que su gestión sea flexible, dinámica y eficiente.

Las funciones que deben ejecutar los gestores educativos están contempladas en el Manual Descriptivo de Clase de Puestos del Servicio Civil. Una de ellas, comprende la función de supervisión docente para la evaluación del desempeño, establecida en el artículo 155, incisos a y b. Por ende, según lo estipula el Estatuto del Servicio Civil (1953), supervisar el trabajo y desempeño de los educadores es un deber, una responsabilidad y una función que el gestor tiene que cumplir; el no realizarla adecuadamente implica que los procesos educativos, especialmente los que desarrollan los docentes en las aulas, se lleven a cabo sin control de los mismos, lo que a su vez genera una carencia de información valiosa para la gestión.

Los gestores educativos son los responsables de hacer que las políticas educativas y todos los procesos relacionados con la educación se realicen de la mejor manera en la organización. Su papel multifuncional requiere que desempeñen distintas actividades al mismo tiempo; entre ellas, una de las que destaca, es su función como supervisor del centro educativo.

De esta función, dependerán muchas otras como la planificación, el control y la realimentación. La supervisión que deben realizar los administradores educativos es, quizás, una de las más 
importantes, pues esta encierra diversos aspectos que se complementan entre sí y de los cuales depende que la institución se consolide como un verdadero centro educativo de calidad.

Por lo tanto, la investigación se plantea como objetivos:

\section{Objetivos generales}

- Analizar la gestión del proceso de supervisión docente en el Colegio Dr. Clodomiro Picado Twight, circuito 02 de la Regional de Educación de Turrialba y Jiménez, para la evaluación del desempeño docente.

- Establecer una propuesta para la supervisión docente en el Colegio Dr. Clodomiro Picado Twight, circuito 02 de la Regional de Educación de Turrialba y Jiménez para la evaluación del desempeño docente.

\section{Objetivos específicos}

1. Identificar las características del enfoque de supervisión docente que desde la gestión en el Colegio Dr. Clodomiro Picado Twight, circuito 02 de la Regional de Educación de Turrialba y Jiménez se lleva a cabo para la evaluación del desempeño docente.

2. Determinar las estrategias de supervisión docente en el Colegio Dr. Clodomiro Picado Twight, circuito 02 de la Regional de Educación de Turrialba y Jiménez para la evaluación del desempeño docente.

3. Establecer los factores positivos para el desarrollo profesional a partir del proceso de supervisión docente desde la gestión en el Colegio Dr. Clodomiro Picado Twight, circuito 02 de la Regional de Educación de Turrialba y Jiménez para la evaluación del desempeño docente.

4. Identificar las limitaciones en el proceso de supervisión docente en el Colegio Dr. Clodomiro Picado Twight, circuito 02 de la Regional de Educación de Turrialba y Jiménez para la evaluación del desempeño docente.

\section{Marco teórico}

\section{Educación}

Partiendo desde su sentido filosófico, Dengo (2011) menciona que“... el origen etimológico de la palabra: ex-ducere, educare, significa'sacar de','hacer salir', o sea, 'externalizar'"' (p. 5). Educar es, por lo tanto, extraer de la persona toda su potencialidad, habilidades y destrezas que, de cierto modo, han permanecido ocultas. 
Es por esta razón, que el sujeto y objeto de la educación es el ser humano. Al ser el individuo un ser cambiante, que evoluciona constantemente, la educación también se desarrolla junto con él y se da en todas las etapas, lugares y condiciones de su vida.

Desde otra perspectiva, León (2007) expresa que "la educación es un proceso humano y cultural complejo" (p. 596). Es claro que, para que exista educación se debe contar con dos elementos esenciales: personas y cultura. Estos dos están íntimamente relacionados; uno no existe sin el otro.

Aunque la educación se dirige a colectivos, esta es individual. Así lo entiende León (2007) cuando asegura que “... la educación universaliza, pero también individualiza" (p. 598). En otras palabras, la educación le permite al ser humano adquirir los conocimientos que, según cada país, debe poseer para lograr la movilidad social, pero también busca potenciar las características distintivas de cada individuo que lo hacen un ser único.

La educación se ocupa de formar al ser humano como un ente integral que posee conocimientos teóricos, los cuales son llevados a la práctica mediante distintas competencias que caracterizan a un individuo dentro de un colectivo. Esto le permite desarrollarse y subsistir en un mundo globalizado, donde las fronteras son estrechas y las oportunidades de desarrollo personal y profesional son muchas.

En los centros educativos, velar porque la educación que se brinda sea contextualizada y especialmente de calidad, es responsabilidad de los docentes, pero más allá, es función del gestor de la educación corroborar que los procesos educativos que cada educador desarrolla se estén realizando de la forma adecuada.

\section{Administración}

Partiendo de su concepto más antiguo, es decir, su raíz epistemológica, Torres y Mejía (2006) indican que“... la palabra administración viene del latín administrare (de ad, a y ministrare, servir), con la acepción de gobernar, regir y disponer de bienes" (p. 114).

Este concepto de administración trae consigo implícito componentes como planeación, ejecución y control; por lo tanto, Chiavenato (2001), tomando como base la definición epistemología, crea su propia definición de administración y la plantea como "...el proceso de planear, organizar, dirigir y controlar el empleo de los recursos organizacionales para conseguir determinados objetivos con eficiencia y eficacia" (p. 3).

Entonces, administrar requiere tomar un conjunto de elementos humanos y materiales que interactúen correctamente entre sí para, de esta forma, lograr que la empresa trabaje fluidamente y alcanzar la eficiencia y eficacia. El trabajo del gerente es coordinar o planificar las funciones que estos elementos tendrán que desempeñar, así como controlarlos y evaluarlos.

Por ende, desde un concepto tradicional, la administración implica una estructura jerárquica, donde el que está en lo más alto es el encargado de administrar a sus subordinados. 
Esta administración se basa en el cumplimiento de reglas establecidas que buscan alcanzar puntos de eficiencia altos, lo que se liga al concepto de organismo social.

En síntesis, se puede concebir que la administración requiere los conocimientos técnicos de su ámbito de acción y que quien se desempeñe en ella, tenga las condiciones personales que le permitan unir conocimiento con humanismo para ejercer, de forma exitosa, la administración.

Por estas características particulares que engloban la administración, la educación ha tomado esta ciencia como tal y la ha trasladado a este campo, generándose así, la administración dela educación.

\section{Administración de la educación}

La administración al ser una ciencia tan amplia, ha llegado a incorporarse a otras ciencias y la educación no ha sido la excepción, por lo que se creó la administración de la educación. Esta, según Venegas (2001), debe ser analizada desde dos perspectivas:

- Administración educativa "formal": basada en leyes, reglamentos y documentos que orientan hacia la toma de decisiones bajo directrices previamente establecidas.

- Administración educativa "informal": tiene como centro al ser humano en su diario vivir dentro de la organización en que labora. (párr. 35-36)

La administración de la educación se encarga de hacer que las leyes, reglamentos y directrices se ejecuten y cumplan en el centro educativo; las decisiones que tome el administrador de la educación deben estar basadas en estos documentos. Además, desde la otra vertiente, se enfoca en el individuo como protagonista del quehacer de la administración educativa.

Otra función de la administración educativa, según Venegas (2001), es: “... que en todo centro educativo exista consciencia de lo que ahí se lleva a cabo, con sentido crítico y visión de mejoramiento permanente" (párr. 73).

Es decir, la función de la administración de la educación es crear una cultura fuerte en el centro educativo; esa cultura hace que quienes allí laboren y estudien, se identifiquen con la institución, al punto de querer lo mejor para ella, aportando criticidad hacia lo que se hace y expresando su visión de cómo mejorar continuamente.

En este sentido moderno de la administración educativa, el director o administrador de la educación, según lo apunta Venegas (2001):

... debe ser agente y partícipe medular del cambio, de acuerdo con las contingencias contemporáneas [...] de esta manera, los centros educativos requieren de un estilo de dirección que fomente una cultura de cambio e innovación para poder aspirar a la flexibilidad y dinamicidad que requiere la época. (párr. 78-79) 
Por lo tanto, los administradores educativos se caracterizan por su proactividad, por estar un paso adelante; prever (mirar hacia el futuro) es una virtud necesaria, pero responder (actuar) es más imprescindible. Si se sabe anticipar pero no actuar del modo más adecuado, los resultados no serán los que se esperaban. Ser flexible, abierto al cambio, caracteriza a un administrador educativo moderno.

Con la administración educativa nace la figura del director, el cual cumple la función de supervisar para controlar su personal, en procura de que estos cumplan sus labores a cabalidad, lo cual requiere de agentes líderes dentro de las instituciones educativas que logren guiar el recurso humano hacia una meta en común.

Además, la administración de la educación, por su origen, se basa en un organigrama vertical de jerarquías dentro de un sistema que permite mantener un control sobre los subordinados mediante procesos de supervisión que pueden generar sanciones si las actitudes y desempeño laboral no son las idóneas. Debido a estas particularidades de la administración educativa, es que se inicia por acoplar otro término más humanizado y contextualizado a las organizaciones educativas, el de gestión de la educación o gestión educativa.

\section{Gestión de la educación}

Gestión educativa es un término que, según Martínez (2012) “... se apega más a las realidades y la misión que tiene la institución educativa en sí misma" (p. 16). Puesto que el centro educativo es visto desde todas sus áreas como resultado, la gestión educativa se encarga de acoplar todas ellas para que trabajen en conjunto armoniosamente.

Se entiende por gestión de la educación, de acuerdo con Martínez (2012):

... capacidad de generar una relación adecuada entre la estructura, la estrategia, los sistemas, el estilo de liderazgo, las capacidades, la gente y los objetivos superiores de la organización considerada, así como la capacidad de articular los recursos de que se dispone de manera de lograr lo que se desea. (p. 17)

La gestión de la educación es un concepto más amplio que administración educativa, debido a que es más completa al incorporar elementos que la administración educativa deja por fuera. De hecho, dentro de la gestión educativa existe la administración de la educación como uno de sus componentes. En palabras de Martínez (2012) "... la administración es dentro de la gestión una dimensión en sí misma, es decir un sistema de sistemas..." (p. 17).

La gestión de la educación, por tanto, necesita de un gestory no un administrador educativo, puesto que se deben desempeñar múltiples funciones, incluyendo la parte de administración. Según López (2011), citado por Oquendo (2013), esta función trae consigo “... la capacidad de conducir a los subalternos, es decir, a los miembros que forman parte de la institución educativa, como una organización" (p. 110). El trabajo del gestor es coordinar las habilidades y destrezas de su equipo de trabajo para lograr cumplir con los objetivos previamente establecidos. 
Para Castillero, Díaz, Morales y Pino de Ochoa (2009):

La gestión educativa es una función que coordina y articula las distintas acciones planificadas por un equipo de trabajo que busca hacer del centro educativo una organización que posibilita la consecución de la intencionalidad pedagógica a través de un proceso de participación efectivo de toda la comunidad escolar. (p. 13)

Dentro de la perspectiva de los autores, la gestión educativa se da en las organizaciones, por lo que incluyen al centro educativo como organización debido a las características que posee. Además, esta concepción incluye un componente que otros autores han dejado de lado como lo es la intencionalidad pedagógica, la meta principal de las organizaciones educativas.

La gestión implica la incorporación de todo el recurso humano con el que cuenta el centro educativo, o al menos con aquellos que están mayormente involucrados, llámense estos personal docente y administrativo. Esta visión de la gestión educativa contrasta con la concepción tradicionalista que indica que el director es el único encargado de gestionar la organización y por ende, en quien recae toda la responsabilidad de las acciones que ocurren en el centro educativo.

Gestionar una institución educativa va más allá de dar órdenes a los docentes, las cuales deben acatar y cumplir; no se trata solo de coordinar elementos claves para el desempeño de un centro educativo. Gestionar implica planificación anticipada y contextualizada a la realidad de la organización, tomar decisiones basadas en datos, crear un ambiente laboral sano y acompañar a los docentes en su labor educativa.

Por lo anterior, es que una de las funciones más importantes que debe desarrollar el gestor educativo es la supervisión; pero, al ser los centros educativos organizaciones particulares donde el objetivo final es la formación de personas integrales, guiados por personas (docentes), la supervisión docente es, por tanto, una de las funciones indispensables que debe ejecutar el gestor de la educación.

\section{Acercamiento al concepto de supervisión en educación}

Si se considera la raíz del término supervisión, se tiene que este, de acuerdo con Lobato (2007) procede “... del latín super (sobre) y videre (ver), es decir, mirar desde arriba, mirar desde lo alto. Super-visar tiene que ver con observar las cosas 'a vuelo de pájaro'..." (p. 30). Entonces, supervisar implica la observación desde lo alto (del jefe a los subordinados). Esta terminología coincide con la visión tradicionalista de la supervisión.

Esta visión es incorporada al ámbito educativo, generándose la supervisión en educación. Paralelamente, Castillero et al. (2009) agrega: “... la supervisión / inspección es un medio del que se vale la administración central para que se cumplan las disposiciones legales y tener información de los resultados y comportamientos de los subalternos" (p. 100). La supervisión se concibe como una función estrictamente desempeñada por los administradores de la 
organización y a su vez, esta tiene un carácter de control y castigo si los resultados no se dan como se habían planteado.

En sus inicios, la supervisión educativa se implementó como un requisito legal desde las altas esferas del sistema educativo hacia las partes, con el objetivo de mejorar la calidad de la educación. La función de supervisión se fue trasladando desde lo más general a lo específico, o sea, de los departamentos ministeriales hasta los centros educativos, al tiempo que su conceptualización también se transformaba.

Actualmente, Kisnerman (1999), citado por Mogollón (2006), establece que: “... la supervisión debe entenderse como una asesoría, un seguimiento, un apoyo profesional que se construye con los supervisores en la práctica..." (p. 2). La visión anterior sobre supervisión enfatiza más la parte humana del gestor educativo y su forma de relacionarse con el medio.

Por lo tanto, la supervisión es un proceso dentro de la gestión que es dinámico y que implica, directamente, la interrelación de los actores educativos, quienes buscan el éxito de la organización educativa y consecuentemente, la calidad de la educación.

La supervisión es interpretada como un proceso integral que debe incluir a varias partes, para poder desarrollarla adecuadamente. Esta no está solo en manos del gestor educativo, sino que docentes, estudiantes, así como padres y madres de familia, son copartícipes de la acción supervisora. Supervisar requiere un líder positivo y proactivo que sea capaz de guiar a los demás hacia una meta en común. Como parte de su papel fundamental dentro de la gestión, la supervisión se ha enfocado hacia uno de los elementos más relevantes dentro de las instituciones educativas, los docentes, dándose de esta manera la supervisión docente.

\section{Supervisión docente}

Según Fermín (1980), citado por Martín (2010), define la supervisión docente como:

... un servicio democrático y sugerente de ayuda y asistencia al educador, destinado a lograr el mejoramiento de los resultados del proceso de enseñanza-aprendizaje, que atañe a todas las actividades y a todas las personas que concurren al proceso educativo y que se realiza mediante un trabajo cooperativo. (p. 7)

La supervisión docente en los centros educativos es realizada por el gestor (director). Esta supervisión no es más que un acompañamiento que se le hace al docente en su labor pedagógica. Por lo tanto, requiere que ambos estén en sintonía y comunicación constante.

Al respecto, Rosales (s. f.) expresa: “... la supervisión debe generar las condiciones para que se dé la integración permanente con el docente, lo que conlleva a planear, probar y revisar su quehacer y aportar al proceso educativo" (p. 3). Si a esto se le suma que el propósito de la supervisión escolar es, de acuerdo con Mogollón (2006): “... establecer una unidad de esfuerzos entre las escuelas, a fin de que, en todas ellas, la tarea educativa se desarrolle de la mejor manera" 
(p. 4). La supervisión debe emplearse como un proceso continuo que permita la mejora de la calidad educativa mediante la constante revisión del quehacer docente.

Para llevar a cabo el proceso de supervisión docente, Smyth (1991), citado por Lobato (2007), propone un proceso que “... se articula a través de la secuencia de cuatro acciones (descripción, información, confrontación y reconstrucción)..." (p. 36). El autor continúa exponiendo que, para lograr este proceso, se deben realizar cuatro preguntas fundamentales:

a) ¿Qué es lo que hago?

b) ¿Cuáles son las razones de lo que hago y qué efectos tiene en el alumnado y en la escuela?

c) ¿Cómo he llegado a actuar de esta forma?

d) ¿Cómo podría hacer las cosas de otra manera? (p. 36)

Como se puede apreciar, el proceso de supervisión requiere de la autorreflexión; es por esto, que los autores citados anteriormente, sostienen que la supervisión docente es un trabajo en conjunto: gestores educativos y docentes.

Cortés y Lorente (2011) señalan que, en Latinoamérica, quienes ejercen la función de supervisar se encuentran con tres obstáculos:"... burocratización de su trabajo por el predominio de la función de control, tentación de instrumentalización de la Supervisión por parte de los poderes públicos y detección de un deficiente desarrollo profesional" (p. 2). Además de los obstáculos antes citados, se suma la falta de tiempo que tienen los gestores de la educación para realizar las diversas funciones que deben desempeñar.

En relación con lo anterior, aunque exista voluntad para ejercer, de forma adecuada, la función de supervisión, la burocracia, la influencia de agentes externos y la falta de tiempo, así como la escaza o nula formación para ejercer esta función, obstaculizan enormemente el desarrollo del proceso de supervisión.

A nivel institucional, el director, ejerciendo la supervisión, debe velar porque el docente se sienta realmente acompañado en su labor, mediante la facilitación de materiales didácticos, infraestructura adecuada para el desarrollo de las lecciones, promover las capacitaciones profesionales necesarias para cada especialidad, crear espacios para el desarrollo personalespiritual, entre otras.

Debido a esta concepción del gestor educativo, es que Machado (2001), retomado por Castillero et al. (2009), enlistan las funciones que debe desempeñar el gestor de la educación en cuanto a la supervisión de los docentes. Estas incluyen:

- Preocupación por la capacitación de los docentes con el fin de mejorar su desempeño y el trabajo en equipo. 
- Intercambiar información con los diferentes niveles de la administración: profesores, estudiantes, padres de familia y el resto de la comunidad.

- Establecer políticas bien definidas y con metas claras. Esto en conjunto con profesores y miembros de la comunidad,

- Interactuar con la comunidad escolar y la comunidad local abriendo la escuela a la participación.

- Hacer efectiva la entrega del currículo básico, así como definir y desarrollar las adaptaciones que correspondan de acuerdo con los intereses específicos de la comunidad local. (p. 54)

Se puede notar como el gestor necesita de su equipo de trabajo para desempeñar, correctamente, sus funciones. Al hacerlo de esta forma, el centro educativo está al servicio de la comunidad, pues se incluyen las perspectivas, opiniones, necesidades e ideas de los actores involucrados; además de crear un sentido de pertenencia organizacional más arraigado, lo que a su vez genera una cultura fuerte que trasciende el ámbito laboral.

Sumado a las funciones anteriores, Castillero et al. (2009) establecen las tareas del gestor educativo. Estas son:

... ayudar al subordinado a encontrar satisfacción en el trabajo, lidiar con los conflictos, comunicar elogios y censuras, supervisar, capacitar, promover el auto desarrollo del personal, promover el trabajo en equipo, brindar apoyo emocional, establecer criterios y normas de actuación, estimular el comportamiento de querer alcanzar metas. (p. 17)

Esto se logra mediante la supervisión y que, para desarrollar este proceso, el gestor educativo necesita emplear algunas estrategias de supervisión docente.

\section{Estrategias de supervisión docente}

Para realizar el proceso de supervisión docente, los gestores educativos hacen uso de estrategias (también conocidas como técnicas) que le permiten cumplir con esta función exitosamente.

Uruñuela (2010) puntualiza que por estrategia se debe entender:

... el arte y manera de dirigir un proceso, el conjunto de pasos y reglas que garantizan una decisión óptima en cada momento. Aplicado a la supervisión, sería el arte y manera de llevar a cabo las funciones que le son propias, el conjunto de reglas o pasos a seguir para conseguir el éxito en su función. (p. 5)

Por medio de las estrategias, el gestor de la educación recopila información valiosa que le permite tomar decisiones, considerando las opiniones de los docentes; claro está que el uso 
de una u otra técnica depende, en gran medida, de la personalidad y forma de gestionar del director, pero también del enfoque de supervisión que prevalezca.

Además, el empleo de una u otra estrategia depende, según Rosales (s. f.), “... del objetivo, el problema por atender, la destreza en su manejo, tiempo de que se dispone, características de los sujetos y la institución en cuánto espacio físico, recursos presupuestarios y logísticos" (p. 8).

Asimismo, el gestor debe considerar si la estrategia a emplear, por su funcionalidad, será directa o indirecta. Castillero et al. (2009) enlista las estrategias directas e indirectas: "Directas: visitas, reuniones, entrevistas, observaciones, etc. Indirectas: análisis de documentos, estadísticas, boletines, notas, cuestionarios, informes..." (p. 113).

Sea cual sea la estrategia o estrategias empleadas, estas brindan información valiosa para la gestión del centro educativo, lo que permite tomar decisiones informadas basadas en evidencia que ha sido recopilada mediante el proceso de supervisión. El cómo se realice este proceso dependerá del enfoque de supervisión que el gestor educativo utilice.

\section{Enfoques de supervisión docente}

Para realizar la supervisión docente, los gestores educativos hacen uso de enfoques, los cuales están muy ligados al tipo de gestión que se encuentra en el centro educativo. Así, se tienen enfoques como el tecnológico, el clínico y el crítico, entre muchos otros. Primeramente, se debe tener claro qué es un enfoque. Lavell (2006) lo define:

... una visión particular o colectiva de un contexto o problema y la forma de abarcarlo con

la intencionalidad de intervenir, cambiar y resolverlo. Enfoques, si son consistentes, deben

fundamentarse implícita o explícitamente en un conjunto diverso de conceptos y, por supuesto, tener una definición de los elementos que lo constituye, consistentes con estos. (p. 5)

Un enfoque está sustentado teóricamente, es decir, se basa en una serie de principios, características particulares, procedimientos y actividades que guían el quehacer hacia la práctica.

Uno de los primeros enfoques empleados en la supervisión es el tecnológico. Lobato (2007) explica: "... el término tecnológico expresa el carácter fundamental de este enfoque, es decir, el uso del conocimiento científico, causal y explicativo, para intervenir y cambiar racionalmente la realidad social, educativa o sanitaria" (p. 31). Por lo tanto, este enfoque es muy estructurado y racional, busca formas objetivas de intervenir en los procesos y cambiarlos si es necesario, pero siempre desde un punto de vista muy científico.

Lobato (2007) añade que la supervisión tecnológica está estrictamente ligada con los conceptos de gestión eficaz y desempeño docente eficiente, enfocándose en los resultados finales más que en el mismo proceso. Se trata de un enfoque en cascada, desde los niveles más altos se transmite toda la información hasta los niveles más bajos, donde se ejecutan las acciones 
por mandato. Se mantiene el origen tecnológico del enfoque y se le agrega la característica burocracia. Es por esta particularidad, que a la supervisión tecnológica se le conoce también como supervisión tecnoburocrática.

Para quienes basan su supervisión en el enfoque tecnológico, la mayor parte del tiempo la invierten en trámites burocráticos, dejando poco espacio para el acompañamiento docente. Por esta razón, surge un enfoque que trata de cubrir los vacíos que la supervisión tecnológica dejaba: la supervisión clínica. Lobato (2007) explica:

El término clínico se refiere a la supervisión cercana en una relación de uno a uno, indicando que el supervisor participa en encuentros cara a cara con los futuros docentes, para discutir los sucesos del aula, a los que ambos han asistido, uno como observador y el otro como profesor. (p. 33)

El enfoque clínico de la supervisión aparta la connotación punitiva y burocrática que caracteriza al enfoque tecnológico, a la vez que busca motivar a los actores educativos para que busquen la actualización profesional y el desarrollo personal. Este punto es de suma importancia dentro de la supervisión clínica, puesto que esta se sustenta en valores profesionales.

Estos valores profesionales, fuera de ser fundamento de la supervisión clínica, deben regir todo centro educativo. Además, los valores coinciden en el trabajo en equipo, el compañerismo profesional, la objetividad y la ética de cada uno. Es importante recalcar que la supervisión clínica se basa en el trabajo mutuo entre docente y supervisor (gestor educativo). Lobato (2007) concluye:

... la supervisión clínica no pretende actuar en o sobre los profesionales, sino trabajar con ellos, en una tarea deliberada cuyo propósito se encuentra en articular sus intenciones, observar y recoger información sobre la intervención y analizar dicha información para reconstruir significados y consecuencias de la misma. (p. 34)

El enfoque clínico tiene muy presente que, para que exista un proceso de supervisión adecuado, es necesaria la participación de los implicados en él. Aunque parece que el enfoque de supervisión clínica es completo, hay autores que encontraron aspectos importantes que no se incluían en este enfoque. Por lo tanto, y como respuesta al enfoque clínico, nace el enfoque crítico de la supervisión.

Para diversos autores, este enfoque contempla nuevas perspectivas. Por ejemplo, Lobato (2007) enlista los nuevos parámetros que cubre la supervisión crítica:

- Determinantes culturales, sociales y políticos de la enseñanza.

- Fines y condiciones actuales de la enseñanza y de su organización escolar.

- Las teorías que el profesorado emplea para la comprensión de su práctica educativa.

- Las contradicciones (y razones de su existencia) en la práctica y en la comprensión del docente. 
- El análisis de los condicionamientos estructurales e históricos que están implicados en el cambio educativo y emancipador en la enseñanza.

- Formas de acción educativas liberadoras que puedan adoptarse en la relación supervisora. (pp. 35-36)

Con la incorporación de estos parámetros, la función supervisora que ejerce el gestor de la educación va más allá del aula, ya que se incorporan elementos como factores estructurales, históricos, sociales y culturales donde la organización educativa está ubicada. Igualmente, en este caso, la supervisión requiere de un análisis de los procesos que cada miembro de la comunidad educativa realiza; es decir, la supervisión va de la mano con la autorreflexión del desempeño de cada actor.

Por su parte, González (2007) propone un enfoque de supervisión escolar que “... articule la gestión supervisora de forma integrada a los procesos técnicos, administrativos, sociales, de asistencia y mediación recientemente denominada como asesoramiento pedagógico integral..." (p. 12).

Este concepto acrecienta la necesidad de un acompañamiento por parte del director de la organización educativa hacia los docentes. En este enfoque, los directores son una especie de asesores pedagógicos quienes deben "... suministrar sugerencias y ayudas constructivas con el propósito de orientar el trabajo administrativo en las instituciones, estableciendo unidades de esfuerzos en las escuelas que ayuden al docente a subsanar debilidades, ejerciendo un liderazgo de carácter democrático y participativo" (González, 2007, p. 13). Se trata de una supervisión docente participativa.

\section{Desempeño docente}

Una de las tareas fundamentales, objeto de la supervisión por parte del gestor educativo, es la relacionada con el desempeño docente, un término amplio que contempla varios aspectos. Es así como Hunt (2009), parafraseando a Dunkin (1997), afirma que:

... la efectividad docente es una cuestión referida a la capacidad del mismo para lograr los efectos deseados sobre los estudiantes [...] la competencia docente como el conocimiento y las habilidades necesarias, y del desempeño docente como la forma en que este se conduce durante el proceso de enseñar. (p. 5)

Por lo tanto, si el docente es competente en sus funciones o roles, su desempeño será bueno; de lo contrario, el desempeño será deficiente. Para Hunt (2009), la competencia docente está directamente vinculada con uno de los roles de los docentes, la práctica pedagógica. La tarea primordial del docente es la relacionada con el área pedagógica, la que se encarga de la formación integral de los estudiantes mediante el empleo de diversas técnicas pedagógicas; 
pero también, la que se encarga de la parte humana de la educación, el desarrollo de habilidades, destrezas, pensamiento crítico, creatividad, valores, entre otros aspectos. Pero el desempeño o rol del docente no queda a nivel de aula.

Por lo tanto, es importante para el gestor de la educación tener claro cuáles son los principales roles que deben desarrollar los docentes que luego se verán reflejados en su desempeño. Es por esto, que Castillero et al. (2009) los resumen en tres áreas: facilitador del aprendizaje, investigador y promotor de la comunidad.

Finalmente, Martínez (2012) concluye que, en el ejercicio de la práctica docente, esta debe ser "... congruente con los constantes cambios y adecuaciones establecidas en los planes y programas de estudio. Implica capacitación y adiestramiento constante por parte de los directivos, coordinadores y docentes para desarrollar los retos de un medio globalizado y cambiante" (p. 94).

El desempeño docente está vinculado con los procesos pedagógicos que el educador desarrolla, el establecimiento de relaciones interpersonales con los estudiantes, compañeros de trabajo, padres y madres de familia, así como comunidad en general, con el crecimiento personal y profesional que incluye la actualización constante y la investigación continua.

\section{Referente metodológico}

La presente investigación es catalogada como un estudio tipo descriptivo que, de acuerdo con Hernández, Fernández y Baptista (2010) “... buscan especificar las propiedades, las características y los perfiles de personas, grupos, comunidades, procesos, objetos o cualquier otro fenómeno que se someta a un análisis" (p. 80).

Por consiguiente, esta investigación pretende analizar, descriptivamente, la gestión del proceso de supervisión docente que se lleva a cabo en el Colegio Dr. Clodomiro Picado Twight, para la evaluación del desempeño docente. Para ello, se contó con la participación de 21 docentes, el director y subdirector del centro educativo, como fuentes primarias de información. Como fuentes secundarias se tienen: tesis, libros, artículos y reglamentos, así como la información del colegio proporcionada en el diagnóstico institucional.

El instrumento empleado para la recolección de la información es un cuestionario dirigido a docentes, el director y subdirector del centro educativo, el cual fue validado en una población con características similares a los sujetos que conforman parte de la muestra de la investigación.

El instrumento de recolección de la información se organiza en cuatro partes: a) la primera sección se refiere a las características de los enfoques de supervisión docente; b) la segunda parte, a las estrategias empleadas para realizar la supervisión; c) la tercera sección contempla los factores positivos que favorecen el desarrollo profesional a partir del proceso de supervisión docente; d) la cuarta parte, a las limitaciones que se presentan en la institución y que obstaculizan 
el proceso de supervisión docente.

\section{Sistematización y análisis de la información}

En este apartado se presenta la información obtenida a través de la aplicación del instrumento al personal docente y administrativo del Colegio Dr. Clodomiro Picado Twight de Turrialba.

\section{Características del enfoque de supervisión docente}

En la siguiente figura, se presentan los indicadores en los cuales coinciden, tanto el personal docente como administrativo del Colegio Clodomiro Picado Twight, en cuanto a las características del enfoque tecnológico de la supervisión docente.

Con base en la Figura 1, en el centro educativo en estudio, las características presentes con mayor fuerza corresponden al enfoque tecnológico de la supervisión. Por lo anterior, el enfoque tecnológico se centra en los resultados, por lo que requiere de una gestión y un desempeño docente eficiente y eficaz, donde se ejecuten las directrices estipuladas por el MEP. Esto es lo que, según los participantes docentes y administrativos, se realiza en el centro educativo.

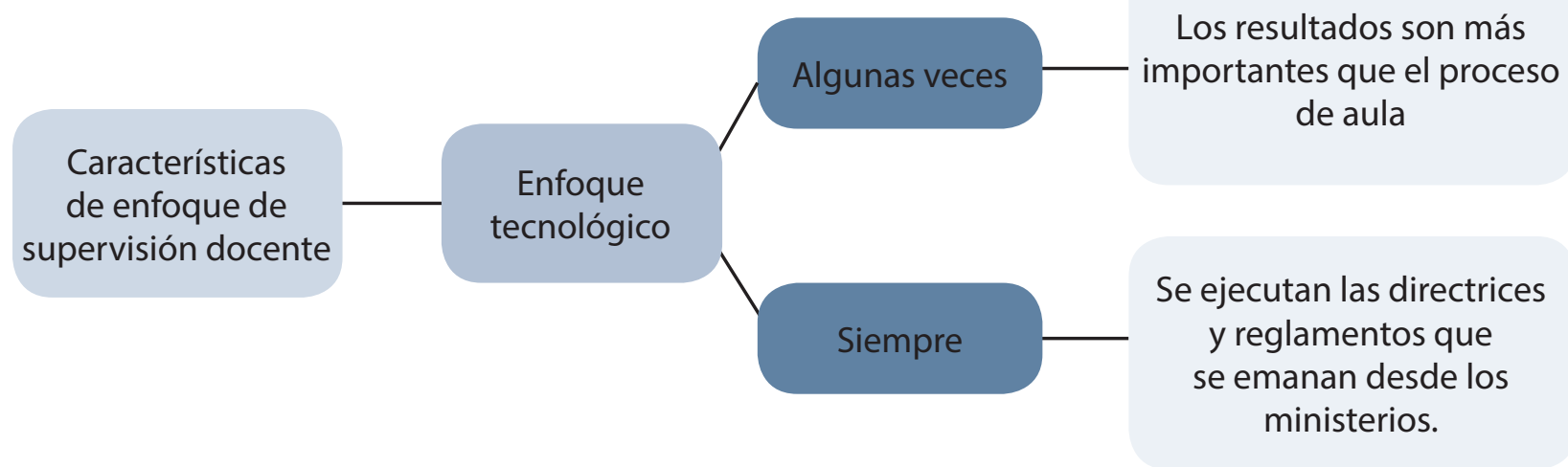

Figura 1. Características de los enfoques de supervisión docente presentes en el colegio Dr. Clodomiro Picado Twight de Turrialba, según la opinión de los docentes, director y subdirector

Nota: Elaboración propia a partir de la información suministrada por el personal docente y administrativo del Colegio Dr. Clodomiro Picado Twight, Turrialba, 2014.

En este orden de ideas, Lobato (2007) puntualiza que la supervisión tecnológica“... funciona como estructura de transmisión y reproducción de conocimiento, focalizada en la transmisión de competencias técnicas singulares [...] o la microsupervisión [...], y en la transmisión de 
un conjunto comprensivo de esta..." (p. 31). El sistema educativo público costarricense está centralizado; el enfoque tecnológico se ajusta de mejor forma en ciertos aspectos que otros enfoques, ya que este tipo de supervisión funciona en cascada; es decir, los altos jerarcas del MEP emanan las directrices a seguir, pasan por los asesores nacionales y regionales hasta llegar a los distintos centros educativos donde se deben ejecutar y posteriormente, medir los resultados.

Es importante tener presente que, si bien es cierto, características de otros enfoques de supervisión docente pueden ser aplicados, el enfoque predominante en la organización en estudio se acopla a las exigencias del sistema educativo, o mejor dicho, a las demandas del MEP. Por cuanto, la gestión se considera eficiente al cumplir con estas exigencias al lograr elaborar y entregar la documentación que el MEP exige, siguiendo un enfoque de supervisión que le permite cumplir las tareas al tiempo establecido.

Por tanto, los enfoques de supervisión están orientados a articular los elementos claves de una organización educativa para que estos funcionen armoniosamente, en pro de la calidad de la educación.

\section{Estrategias de supervisión docente}

En cuanto a las estrategias de supervisión docente (esenciales para llevar a cabo este proceso), se da una coincidencia entre lo expresado por los docentes participantes, el director y subdirector de la institución. Esta concordancia se ilustra mejor en la figura que se presenta a continuación.

La Figura 2 muestra que, en la organización objeto de estudio, la principal estrategia que se da para la supervisión docente y en la cual concuerdan el personal docente y administrativo, son las reuniones de departamento; es decir, una estrategia directa.

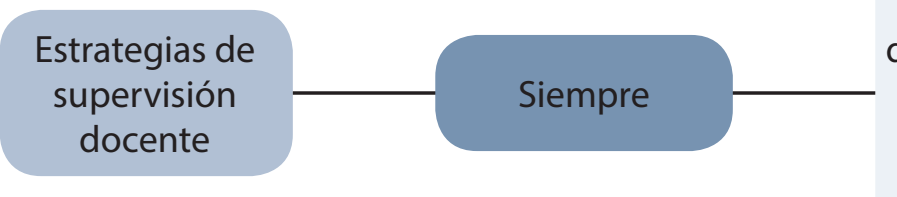

Realización de reuniones de departamento con el fin de mantener informada a la gestión acerca de los procesos pedagógicos que cada docente de área realiza.

Figura 2. Estrategias de supervisión docente presentes en el colegio Dr. Clodomiro Picado Twight de Turrialba, según la opinión de los docentes, director y subdirector

Nota: Elaboración propia a partir de la información suministrada por el personal docente y administrativo del Colegio Dr. Clodomiro Picado Twight, Turrialba, 2014. 
Las estrategias de supervisión docente son de suma importancia ya que, según lo estipula el Ministerio de Educación Pública de Perú (s. f.), "... ayudan a unificar y armonizar las actividades del personal, así como, intercambiar ideas sobre aspectos que posibiliten mejorar el proceso enseñanza-aprendizaje" (p. 26).

En dichas reuniones se tratan distintos aspectos o temas de interés; estas tienen validez y son del dominio de los miembros que participan de la reunión. Mediante las reuniones, la gestión se garantiza que la información fluya entre las partes interesadas y se evita posibles conflictos por interrupciones en la comunicación.

Las reuniones permiten, tanto al personal docente como administrativo, lograr múltiples propósitos en un solo lugar y en un tiempo relativamente corto; esto a su vez, propicia un ambiente institucional sano al darse una comunicación directa, se evitan malos entendidos, se toman acuerdos en conjunto y en especial, se mantiene la gestión del centro educativo al tanto de los acontecimientos que se dan en las aulas, lo que conduce hacia un centro educativo de calidad.

\section{Factores positivos para el desarrollo profesional a partir del proceso de supervisión docente}

Al comparar las opiniones de docentes y personal administrativo, los educadores y el director coinciden en solo un indicador en este aspecto de factores positivos para el desarrollo profesional. Este se ve reflejado en la Figura 3.

La Figura 3 muestra cómo en el centro educativo, a raíz del proceso de supervisión docente, la gestión busca apoyar a los docentes mediante charlas, talleres y capacitaciones.

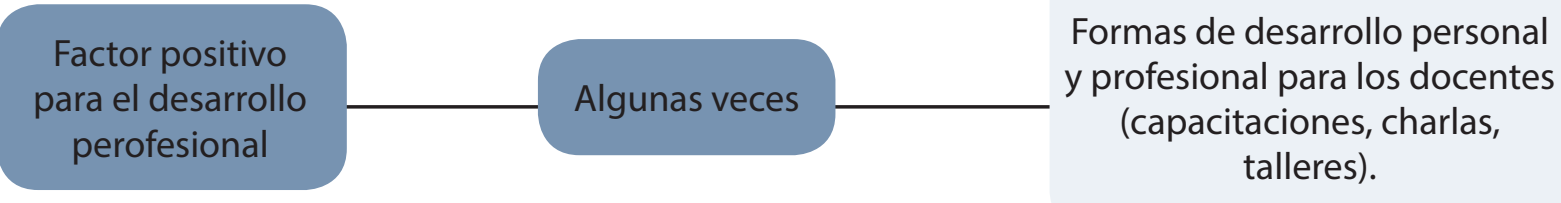

Figura 3. Factores positivos que favorecen el desarrollo profesional a partir del proceso de supervisión docente en el colegio Dr. Clodomiro Picado Twight de Turrialba, según la opinión de los docentes, director y subdirector

Nota: Elaboración propia a partir de la información suministrada por el personal docente y administrativo del Colegio Dr. Clodomiro Picado Twight, Turrialba, 2014. 
Este proceso de búsqueda por parte de la gestión para apoyar al personal docente, es una de sus tareas a cumplir; específicamente es una tarea administrativa que, de acuerdo con Castillero et al. (2009) “... implican la dirección de personal, que incluye adiestramiento, asesoría y desarrollo de personal" (p. 101). Una de las tantas tareas que tiene el director que realizar cuando supervisa es, precisamente, buscar la forma en que puede brindar a los docentes mecanismos para que estos se mantengan actualizados.

El director, utilizando la supervisión como fuente rica de información, puede ayudar a su personal no solamente a desarrollarse más y mejor como profesional, sino también como ser humano pensante y que siente. Al final, esto es un conjunto; no se puede ser docente sin ser persona.

Estos deben estar en constante actualización; para Martínez (2012) un docente debe ser “... congruente con los constantes cambios y adecuaciones establecidas en los planes y programas de estudio. Implica capacitación y adiestramiento constante por parte de los directivos, coordinadores y docentes para desarrollar los retos de un medio globalizado y cambiante" (p. 94).

En este sentido, es que la gestión de supervisión que realiza el director promueve el desarrollo profesional de quienes llevan a cabo el proceso de enseñanza aprendizaje, desde el acompañamiento y la capacitación continua. Los gestores educativos son los responsables directos de velar porque sus colegas de trabajo cuenten con los conocimientos, habilidades y destrezas necesarias para ejercer la profesión docente, aspectos que deben posteriormente ser trasladados y puestos al servicio del centro educativo. A esto, se le une las exigencias que cada comunidad le demanda a la organización educativa, en procura de una mayor calidad educativa.

Por lo tanto, el desarrollo profesional es una obligación de los docentes y los gestores educativos. Suárez (2005), citado por Barrantes, Céspedes, Mejía, Picado, Rojas y Zúñiga (2010), indica que el desarrollo profesional docente “... debe considerarse como un proceso inherente a la carreta docente, vinculado al desarrollo institucional y como una estrategia para el cambio global" (p. 4). Dentro de la planificación anual que realiza el gestor junto con el personal administrativo y docente, se deben contemplar actividades de desarrollo profesional y personal que potencien el centro educativo hacia la mejora continua y que lo convierta en una organización competente en su contexto.

\section{Limitaciones en el proceso de supervisión docente}

Si se contrasta el punto de vista de los docentes, el director y subdirector, se encuentra una concordancia en relación con los factores limitantes que se presentan en la organización educativa y que obstaculizan realizar procesos de supervisión docente. Este factor se ilustra en la figura a continuación. 


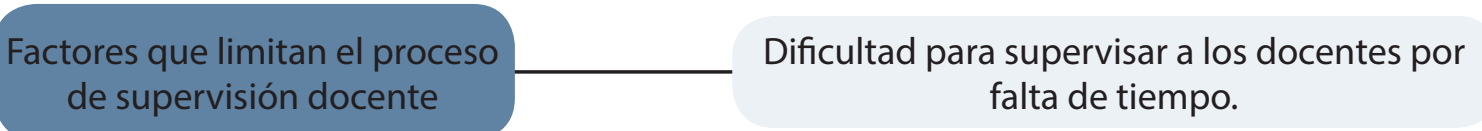

Figura 4. Factores que limitan el proceso de supervisión docente en el colegio Dr. Clodomiro Picado Twight de Turrialba, según la opinión de los docentes, director y subdirector

Nota: Elaboración propia a partir de la información suministrada por el personal docente y administrativo del Colegio Dr. Clodomiro Picado Twight, Turrialba, 2014.

La Figura 4 ejemplifica cómo el factor tiempo obstaculiza el proceso de supervisión docente, el cual es percibido tanto por los educadores como por el personal administrativo. Esta limitante de tiempo se da porque el gestor tiene que ejercer distintas funciones que, en muchas ocasiones, tienen un grado mayor de urgencia por cumplir; tal es el caso de la confección y entrega de documentación que se les solicita por parte del MEP, según lo manifiesta el personal administrativo del centro educativo.

Además, sobresale que este factor es uno de los que aquejan con mayor incidencia a las organizaciones educativas a nivel latinoamericano. Lo anterior, sustentado por Cortés y Lorente (2011) quienes señalan que, en Latinoamérica, los encargados de realizar funciones de supervisión se encuentran con tres obstáculos: "... burocratización de su trabajo por el predominio de la función de control, tentación de instrumentalización de la Supervisión por parte de los poderes públicos y detección de un deficiente desarrollo profesional" (p. 2).

El cumplir procesos engorrosos, por parte del personal administrativo, significa una fuerte inversión de tiempo que impide a la gestión ejecutar estrategias de supervisión más constantes. La alta burocratización del sistema educativo costarricense es el obstáculo más grande al que se enfrentan quienes laboran en él. En consecuencia, realizar una supervisión docente como acompañamiento, se convierte en un reto casi inalcanzable para la gestión del centro educativo.

\section{Conclusiones}

La investigación permitió identificar el enfoque de supervisión docente predominante por la gestión, las estrategias empleadas para realizar dicha función, así como los factores positivos que se desprenden de esta y las limitantes a las que se enfrenta la gestión al realizar este proceso de supervisión.

En relación con las características del enfoque de supervisión docente aplicado desde la gestión del centro, se considera que, en la institución en estudio, sobresale la presencia del enfoque tecnológico y clínico, y se nota la ausencia del enfoque crítico. Esta ausencia de ciertas características típicas y esenciales del enfoque crítico no es compartida por el personal administrativo de la institución, quienes aseguran que estas sí se encuentran presentes. Se 
evidencia, por tanto, como existe una discrepancia entre el enfoque de supervisión que percibe y vive el personal docente con el que asegura la administración se lleva a la práctica.

Desde la gestión, la implementación de este enfoque recalca la necesidad de un cambio a nivel nacional de los procesos de supervisión que se realizan desde las altas esferas del MEP, pasando por las direcciones regionales de educación hasta el núcleo de cada centro educativo, donde la práctica docente se ve afectada por la falta de acompañamiento pedagógico que un enfoque de supervisión más democrático y participativo pueda brindarle y por lo tanto, mejorar cualitativamente la calidad de educación que la sociedad demanda.

En relación con las estrategias de supervisión docente, se concluye que estas son indispensables para realizar procesos de supervisión integrales, los cuales están ausentes; la utilización de una lista de cotejo o cualquier otro instrumento en las observaciones de aula y la aplicación de cuestionarios al personal docente o estudiantes para evaluar el desempeño de los educadores, no se da. Por lo tanto, es necesario fortalecer estos aspectos que favorecen, en gran medida, la supervisión docente participativa y democrática, que incentiva a la reflexión de los actores educativos y propician servicios educativos de calidad.

Asimismo, se necesita ampliar y actualizar dentro del personal docente y a los mismos administradores educativos, lo que la supervisión implica y verla más allá de una observación de clase, haciendo uso de distintas estrategias de supervisión docente que permiten acompañamiento continuo entre los mismos educadores y del gestor hacia los docentes, tarea que debe iniciar por el mismo director.

En cuanto a los factores positivos que favorecen el desarrollo profesional docente a partir del proceso de supervisión, se concluye que en el centro educativo se necesita motivar a los educadores mediante el reconocimiento de las acciones sobresalientes que realizan docentes que benefician el proceso de enseñanza aprendizaje y que buscan mejorar la calidad de la educación que la organización ofrece. Es necesario que la gestión realice los trámites necesarios para brindarle a los educadores mayores oportunidades de actualización constante en temas propios de la asignatura, así como en aspectos relacionados con mediación pedagógica, evaluación de los aprendizajes, resolución de conflictos, adecuaciones curriculares, entre otros elementos claves para un buen desempeño docente.

Refiriéndose a las limitaciones que pueden obstaculizar el proceso de supervisión docente, se concluye que, a pesar de que existe una clara limitante de tiempo, en el centro educativo no se presentan otros factores que pueden ocasionar una obstaculización hacia la supervisión docente. Por lo tanto, la gestión debe buscar mecanismos o alternativas que contribuyan a minimizar la limitación que el manejo del tiempo representa, mediante la aplicación de un enfoque de supervisión docente que le permita realizar este proceso de forma constante, propicie el desarrollo de los docentes como profesionales y beneficie la calidad de educación que la organización educativa ofrece. 
Para minimizar el efecto negativo que este aspecto tiene sobre la supervisión docente, específicamente en las observaciones de aula, el gestor puede recurrir al coordinador académico de la institución, quien tiene lecciones asignadas para esta labor y así, designarle las visitas de aula que deben realizarse a los educadores; por tanto, el tiempo es un factor manejable para la gestión.

En síntesis, en el centro educativo se debe procurar realizar una gestión de excelencia basada en un enfoque de supervisión contextualizado, democrático y participativo, que propicie procesos educativos de calidad acordes a las exigencias que la sociedad demanda de la educación. La visión de supervisión que se tiene actualmente debe cambiarse por una más integral que incluye a todos los actores educativos, tarea que debe realizar el gestor por ser él la cabeza de la institución y el modelo más cercano a seguir.

\section{Propuesta de intervención}

A raíz de los hallazgos encontrados por medio del estudio, se presenta una propuesta de intervención que busca apoyar y guiar a los gestores educativos en su labor, específicamente la función de supervisión docente que debe realizar si se desea mejorar los espacios educativos en la organización educativa y por ende, la calidad de la educación que la institución ofrece.

La meta principal es apoyar la gestión del centro educativo en los procesos de supervisión docente, para que estos generen un impacto positivo que fomente el crecimiento profesional y personal de los miembros de la organización y que, a su vez, promueva espacios de participación y reflexión del personal. Esto se da mediante la ejecución de tres planes de acción que contemplan aspectos de un enfoque de supervisión participativo y democrático.

Para ello, se propone el diseño de un plan de acción que promueva un enfoque de supervisión democrático y participativo en el Colegio Dr. Clodomiro Picado Twight de Turrialba, cuyos objetivos específicos pretenden:

- Promover la concientización relacionada con los procesos de supervisión democráticos y participativos en el Colegio Dr. Clodomiro Picado Twight de Turrialba.

- Fomentar procesos de supervisión democráticos y participativos en el Colegio Dr. Clodomiro Picado Twight de Turrialba.

- Elaborar un conjunto de instrumentos de evaluación que permita medir los procesos de supervisión democráticos y participativos, como herramienta para la gestión en el Colegio Dr. Clodomiro Picado Twight de Turrialba.

- Desarrollo de la propuesta

La propuesta de intervención propone integrar a la comunidad educativa (gestor educativo, docentes y estudiantes) para implementar un proceso de supervisión democrático y participativo, por medio de la ejecución de tres planes de acción que se resumen en la siguiente figura. 


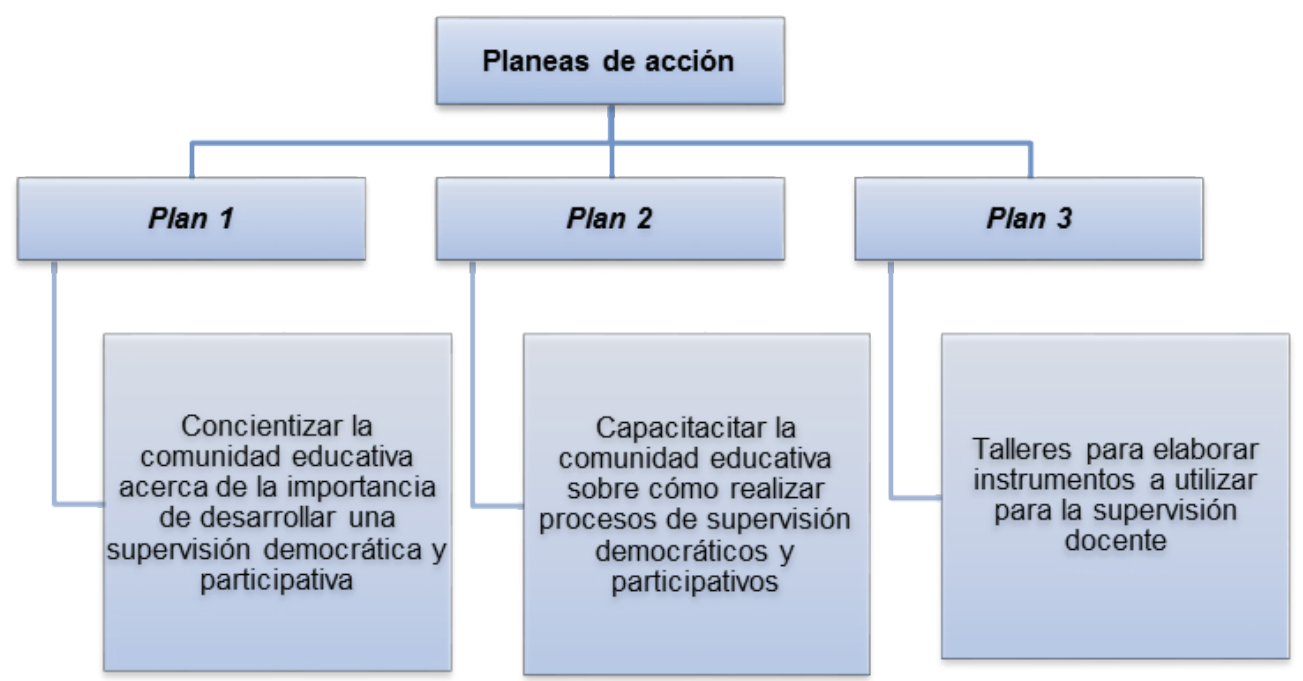

\section{Figura 5. Planes de acción a desarrollar para una supervisión participativa y democrática}

Nota: Elaboración propia a partir de los planes de acción realizados en la propuesta de intervención, 2014.

Los planes de acción están basados en la participación activa de docentes, estudiantes y personal administrativo quienes, en conjunto, deben trabajar para realizar un proceso constante de supervisión docente participativo y democrático, mediante el uso de instrumentos de evaluación del desempeño docente elaborados por los mismos actores educativos en los talleres. Los resultados obtenidos por educadores, estudiantes y gestor educativo se unificarán para lograr una evaluación integral del desempeño de los docentes.

\section{Referencias}

Barrantes, A., Céspedes, X., Mejía, A., Picado, S., Rojas, Y. y Zúñiga, A. (2010). La opinión del personal docente en educación primaria sobre las actividades de desarrollo profesional que realiza y la influencia que tienen en su práctica pedagógica. Revista Electrónica Actualidades Investigativas en Educación, 10 (3), 1-15. Recuperado de: http://revista.inie. ucr.ac.cr/uploads/tx magazine/personal.pdf

Castillero, A., Díaz, J., Morales, F. y Pino de Ochoa, L. (2009). Gestión y supervisión en el centro de educación básica. San José, Costa Rica: Coordinación Educativa y Cultural Centroamericana. 
Chiavenato, I. (2001). Administración: proceso administrativo. Colombia: McGraw-Hill.

Cortés, R. y Lorente, A. (2011). La supervisión educativa en América Latina ante las metas educativas de 2021, propuestas por la OEl. Revista Iberoamericana de Educación, 1 (57), 1-10. Recuperado de: http://www.rieoei.org/deloslectores/4601Cortes.pdf

Dengo, M. (2011). Educación costarricense. San José, Costa Rica: EUNED.

Estatuto del Servicio Civil. (1953). Recuperado de: http://www.tse.go.cr/pdf/normativa/ estatutodeserviciocivil.pdf

González, E. (2007). Un modelo de supervisión educativa. Red de revistas científicas de América Latina, el Caribe, España y Portugal, 13 (25), 11-35. Recuperado de: http://redalyc.uaemex. $\underline{\mathrm{mx} / \mathrm{src} / \text { inicio/ArtPdfRed.jsp?iCve }=76111479002}$

Hernández, R., Fernández, C. y Baptista, P. (2010). Metodología de la investigación. México: Mc Graw Hill.

Hunt, B. C. (2009). Efectividad del desempeño docente. Una reseña de la literatura internacional y su relevancia para mejorar la educación en América Latina. Recuperado de: http://www.oei. es/pdf2/documento preal43.pdf

Lavell, A. (2006). Consideraciones en torno al enfoque, los conceptos y los términos que rigen con referencia a la reducción del riesgo y la atención de desastres en los países Andinos miembros del CAPRADE. Recuperado de: http://www.comunidadandina.org/predecan/doc/r1/ docAllan1.pdf

León, A. (2007). Qué es la educación. Red de revistas científicas de América Latina, el Caribe, España y Portugal, 11 (39), 595-604. Recuperado de: http://www.redalyc.org/pdf/356/35603903. pdf

Lobato, C. (2007). La supervisión de la práctica profesional socioeducativa. Revista de Psicodidáctica, 12 (1), 29-50. Recuperado de: https://addi.ehu.es/bitstream/10810/7169/1/Rev.\%20 Psicodidactica\%2012\%281\%29\%20-\%2029-50.pdf

Martín, E. (2010). Supervisión educativa. Recuperado de: http://mail.mec.gov.py/cmsmec/wpcontent/uploads/2010/02/moduloiiia t3.pdf

Martínez, L. (2012). Administración educativa. México: Red Tercer Milenio S.C.

Ministerio de Educación Pública de Perú. (s. f.). Lineamientos y estrategias generales para la supervisión pedagógica. Recuperado de: http://www.minedu.gob.pe/Delnteres/xtras/ download.php?link=supervision pedagogica.pdf

Mogollón, A. (2006). Funciones de la supervisión escolar en Venezuela. Aproximación a un modelo. Revista Electrónica Actualidades Investigativas en Educación, 6 (3), 1-22. Recuperado de: $\underline{\text { http://revista.inie.ucr.ac.cr/uploads/tx magazine/funcio.pdf }}$ 
Oquendo, C. (2013). Gestión del gerente educativo en el acompañamiento docente en las escuelas rurales de la parroquia Altagracia, Municipio Miranda, Estado Zulia. Centro de Investigación de Ciencias Administrativas y Gerenciales, 11 (2), 107-118. Recuperado de: http://publicaciones.urbe.edu/index.php/cicag/article/view/2241/4053

Rosales, R. (s. f.). Algunas reflexiones sobre la supervisión como proceso educativo. Recuperado de: www.ts.ucr.ac.cr

Torres, S. y Mejía, A. (2006). Una visión contemporánea del concepto de administración: revisión del contexto colombiano. Cuadernos de Administración Bogotá, 19 (32), 111-133. Recuperado de: http://cmapspublic2.ihmc.us/rid=1232641753171 1848515022 2944/ ADMINISTRACION.pdf

Uruñuela, P. (2010). La supervisión externa de las instituciones escolares. Modelos, técnicas $e$ instrumentos. Recuperado de: http://www.mec.gov.py/cmsmec/wp-content/ uploads/2010/02/moduloiiia t8.pdf

Venegas, P. (2001). Retos y perspectivas de la administración educativa en un nuevo milenio. Recuperado de: http://www.euned.ac.cr./.../La\%administraci\%C3\%B3n\%20Educativa\%20 en20\%el\%20Siglo\%XXI 